\title{
Let's mobilise the secret army
}

\section{Susan Quilliam}

\section{Introduction}

Here's a question. What if you had at your back an army of trained helpers who could support your work by identifying sexual difficulties in patients, helping to resolve those difficulties, then referring on to you when they couldn't achieve such resolution. How wonderful would that be?

Well actually you do have such an army, although neither you nor the army itself may be aware of its existence. Because there are many thousands of medical professionals who are in a position to spot sexual problems and get them sorted, were they resourced to do so.

\section{Non-sexual health practitioners as triage}

I'm talking about primary and secondary care nurses and doctors who are, whether they realise it or not, dealing with patients whose illnesses impact on their sex lives. For example, a cardiovascular specialist will almost certainly deal with many who, as a direct result of their condition, suffer problems of arousal, erection, orgasm - or simple uncertainty about whether it is safe to make love. The musculoskeletal specialist, the physiotherapist and the osteopath will almost certainly have patients whose pain and immobility have a serious impact on their sexual functioning. The oncologist will have patients who not only physically find it difficult to make love, but post-treatment are traumatised about doing so. The gastrointestinal specialists will be seeing patients whose erotic motivation has plummeted because of their colostomy bag; the spinal injury specialists will be seeing patients who long to have sex but whose response is so limited as to make it either impossible or pointless. And that's without even considering the many professionals treating conditions that don't themselves trigger sexual problems but require the prescription of medications that do.

\section{Most general medical professionals don't see, read and speak 'sex talk' as a regular part of their job.}

If all these 'non-sexual practitioners', as we might call them, made sexual functioning a regular part of their consultation, there would not only be a hugely positive impact on the work of those in the front line of sexual trouble shooting but, far more importantly, there would be a hugely positive impact on patient quality of life.

Once these professionals had spotted a difficulty, they could as a first step use their own specialist knowledge to help solve that difficulty; maybe defining what level of sexual activity is safe, maybe easing pain, maybe altering medication, maybe suggesting ways round practical issues such as restricted movement or lack of sensation. For issues outside their focused expertise, they could then refer on to urinary/gynaecological consultants or psychosexual therapists.

J Fam Plann Reprod Health Care 2010; 36(4): 249-250

\section{Cambridge, UK}

Susan Quilliam, BA, Cert Ed, MNLP, Freelance Writer, Broadcaster and Agony Aunt

Correspondence to: Ms Susan Quilliam.

E-mail: susan@susanquilliam.com

\section{Sexual symptoms as diagnostic tools}

But is there anything in it for the non-sexual practitioners? Absolutely. Because what if the professionals in the areas I've outlined above - such as oncology, endocrinology, cardiology - had a whole new bank of diagnostic aids to support their work in identifying medical problems, then diagnosing and treating their patients? Would they not jump at the chance? Well they do have such bank of aids the sexual problems themselves.

\section{Not every practitioner will realise how important it is for them to include sexual functioning in their practice.}

Because however endlessly I write in this column about the crucial role that emotions play in sex, I know all too well that a sexual problem may be down to purely medical triggers. Erectile dysfunction may not be down to 'old age' but to diabetes. Loss of desire may not be a sign of a 'failing marriage' but of a failing thyroid gland. Were the non-sexual practitioner to regularly enquire about a patient's sexual functioning then they might gain valuable added information relevant to making the diagnosis and to providing treatment.

\section{Sexual advice as medical intervention}

No, don't panic. I'm not suggesting that every time a patient walks into the consulting room they get asked for graphic details of their erotic adventures. But I am suggesting that just as a consultation might include questions about shifts in pain or fatigue levels, so it could include questions about shift in sexual activity - both might give clues to a medical problem or to patient progress. And just as any follow-up care programme might cover recommendations about diet, mobility and medication regimes, so it could cover recommendations on whether lovemaking can take place, and advice on how best to achieve that given the illness, surgery or treatment the patient has had.

Non-sexual practitioners spotting sexual problems and helping solve them? Sexual problems used as a diagnostic tool for medical conditions? Sexual well-being as part of a long-term treatment and maintenance programme? The term 'integrated health' hasn't always had a good press, so I am wary of mentioning it. But that's essentially what we are talking about here.

\section{We need to reach a stage where \\ all practitioners see positive sexuality as an integral part of diagnosis, treatment and general health care.}

Because perhaps a patient's sexual functioning is every bit as medically significant as their respiratory, cardiac and muscle functioning when it comes to diagnosis. And perhaps a patient's sexual satisfaction is as important as their diet and exercise regime when it comes to physical and mental recovery. And - I'll go further - perhaps patients' sexual satisfaction (not just their contraception use or their avoidance of sexually transmitted infections) is just as vital to consider as their smoking or drinking habits when it comes to general health. 


\section{Sexual discussion as consultation}

At this point, of course, we come up against hard reality. Because I know that the vision I've outlined above - to have all health professionals actively considering happy sexual functioning as part of their patient's good health will be tricky to achieve.

On a practical level, I'm very aware that it is difficult even for sexual specialists like ourselves to initiate conversations about a client's love life; how much more difficult, then, for the general medical professional to dive in? Most don't see, read and speak 'sex talk' as a regular part of their job. They don't have experience of normalising such conversations for their patients. They may feel utterly de-resourced to advise practically (more challengingly still, to support emotionally.) They may have no idea how, or where, to refer on.

\section{Helping patients who want to be sexually active to be so is a vital part of any medical care.}

And even if the practicalities of enrolling nonspecialists were solved, there may still be an insurmountable belief barrier to overcome. Not every practitioner will realise how important it is for them to include sexual functioning in their practice. They may feel that sex is 'an optional extra' in a patient's life. (And yes, for many patients it is; many patients neither want nor need a regular love life and I would be the first to argue that that is their choice, and not their failure.)

\section{Sexual activity as a lifestyle marker}

But I would also be the first to argue that for many patients having a regular, loving sex life is hugely important. For most, losing desire means they lose self-esteem; becoming non-functional means they become deskilled, often depressed. They may feel unable to turn to their partner even for simple affection, for fear that they then won't be able to perform; in the end they feel not only unloved and unlovable but also unloving. And this in the context of already suffering the physical pain and emotional debilitation that illness - particularly chronic illness brings. Can there be any doubt that helping patients who want to be sexually active to be so is a vital part of any medical care?

In short, I would argue that if we want to mobilise nonsexual specialists as I'm suggesting, the most crucial step is to get our entire profession to acknowledge the vital role that sexuality plays in health. We need to move beyond regarding sex as something only specialist sexual health professionals pay attention to, and then only when it goes

\section{Box 1: Enrolling non-specialists}

If what I have proposed strikes a chord with you, then spread the word in the following ways:

- Help non-sexual colleagues realise how important sexuality is in diagnosis, treatment and general health care.

- Support colleagues by offering information and resources about how to include sexual functioning in their practice.

- Copy colleagues in on the guidelines for raising sexual issues (see Box 2).

- Help colleagues identify local practitioners to whom they can refer patients with sexual problems that they themselves cannot resolve.

Box 2: Raising sexual issues with patients during consultation: suggested guidelines

When formulating the questions:

- You may want to ask about these sexual problems that can indicate physical problems: fading desire, erectile dysfunction, inability to allow penetration, inability to ejaculate, inability to orgasm, pain on intercourse, immobility or discomfort during sexual movement, negative body image.

- It is particularly useful to ask questions about any change in sexual activity. "Have you noticed any difference in ... your desire for sex ... your erection?" as this can indicate a shift in health.

When beginning the consultation:

- Flag up the issue well before you ask any sexual questions. "I may ask you questions that seem strange/out of context, but they will help me to help you."

- Normalise the process. "These are questions that I ask all my patients."

- Provide a way forward on patient negative response. "Tell me if any question makes you feel uncomfortable and we'll find a way to talk about it that helps you feel more at ease."

When asking the questions:

- Be relaxed. The patient will take their lead from you about whether to be tense or nervous about the questions.

- Use clinical rather than informal terms to begin with, but if the patient seems confused, ask them what terms they would use for the same body part or activity; then if you feel comfortable with their term, use that

- Validate the patient, so reinforcing their willingness to answer such questions next time. "Your answers were really useful. Thank you."

wrong. We need to reach a stage where all practitioners see positive sexuality as an integral part of diagnosis, treatment and general health care (see Boxes 1 and 2 for some suggested steps towards such a scenario).

We need to widen the definition of 'health care' to include helping patients have as positive and active love life as they wish - whatever their injury, whatever their condition, however well or ill they may be.

Statements on funding and competing interests Funding None identified. Competing interests None identified.

\section{BOOK REVIEW}

Abnormal Uterine Bleeding. Malcolm G Munro. New York, NY: Cambridge University Press, 2010. ISBN-13: 978-0-52184-849-7. Price: $£ 35.00$. Pages: 251 (paperback)

This compact clinical guide gives an up-to-date overview of the common gynaecological complaint of abnormal uterine bleeding. It is immediately apparent that the author, Malcolm Munro, has real enthusiasm for his speciality and a genuine desire to improve patient care. The book is divided into five sections: background, anatomy and physiology, classification and pathogenesis, clinical management, and procedures. The introductory historical context provides an entertaining and informative insight into how far we have progressed in the understanding of the pathogenesis and treatment of abnormal bleeding. Clear illustrations and diagrams guide the reader through the complexities of the anatomy and physiology of menstruation. Munro addresses the inconsistencies in nomenclature in relation to abnormal bleeding and provides a useful summary of the internationally agreed new PALM-COEIN classification system. The clinical management section provides a practical evidence-based approach with useful flow charts to aid physician decision-making. The book proceeds to describe common gynaecological procedures used in the treatment of abnormal uterine bleeding. This section is fantastically supplemented with an inclusive DVD of real-time procedures with helpful commentaries. As for the negative aspects, the book does take some serious reading as there is abundant, albeit interesting, prose. A clinician seeking a quick reference guide would probably be better off looking elsewhere. But for those with a specialist interest in abnormal uterine bleeding, this book is definitely worth the effort.

Reviewed by Jackie Maybin, BSc, MBChB Clinical Research Fellow in Obstetrics and Gynaecology, University of Edinburgh, Edinburgh, UK 